This item was submitted to Loughborough's Research Repository by the author.

Items in Figshare are protected by copyright, with all rights reserved, unless otherwise indicated.

\title{
Advanced computer-aided design for bone tissue-engineering scaffolds
}

PLEASE CITE THE PUBLISHED VERSION

PUBLISHER

(C) IMechE / Professional Engineering Publishing

VERSION

VoR (Version of Record)

LICENCE

CC BY-NC-ND 4.0

REPOSITORY RECORD

Ramin, Ettore, and Russell A. Harris. 2019. "Advanced Computer-aided Design for Bone Tissue-engineering Scaffolds". figshare. https://hdl.handle.net/2134/4622. 
This item was submitted to Loughborough's Institutional Repository (https://dspace.lboro.ac.uk/) by the author and is made available under the following Creative Commons Licence conditions.

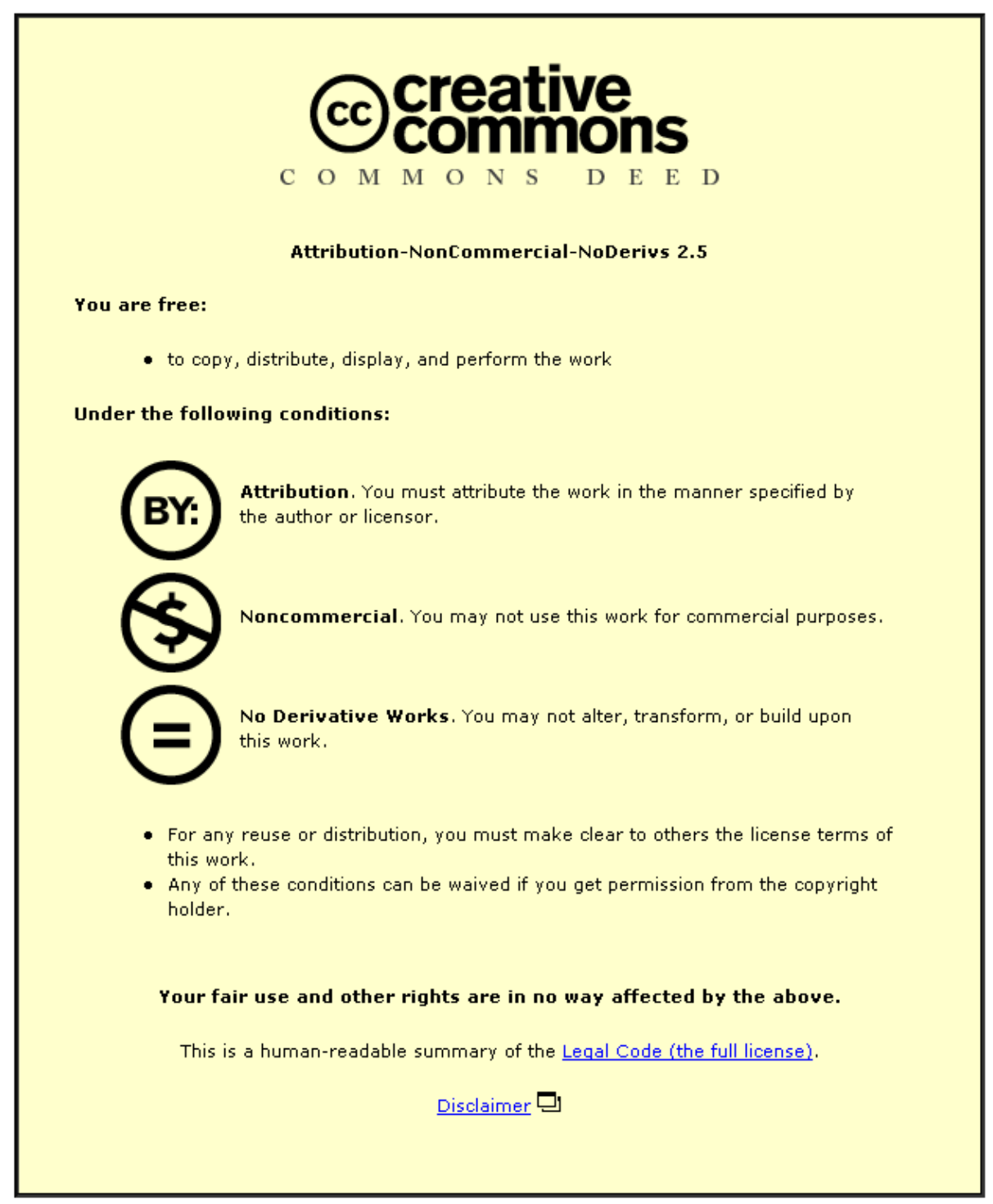

For the full text of this licence, please go to: http://creativecommons.org/licenses/by-nc-nd/2.5/ 


\title{
Advanced computer-aided design for bone tissue-engineering scaffolds
}

\author{
E Ramin* and R A Harris \\ Wolfson School of Mechanical and Manufacturing Engineering, Loughborough University, Loughborough, UK
}

The manuscript was received on 19 May 2008 and was accepted after revision for publication on 24 November 2008.

DOI: 10.1243/09544119JEIM452

\begin{abstract}
The design of scaffolds with an intricate and controlled internal structure represents a challenge for tissue engineering. Several scaffold-manufacturing techniques allow the creation of complex architectures but with little or no control over the main features of the channel network such as the size, shape, and interconnectivity of each individual channel, resulting in intricate but random structures. The combined use of computer-aided design (CAD) systems and layer-manufacturing techniques allows a high degree of control over these parameters with few limitations in terms of achievable complexity. However, the design of complex and intricate networks of channels required in CAD is extremely time-consuming since manually modelling hundreds of different geometrical elements, all with different parameters, may require several days to design individual scaffold structures.

An automated design methodology is proposed by this research to overcome these limitations. This approach involves the investigation of novel software algorithms, which are able to interact with a conventional $\mathrm{CAD}$ program and permit the automated design of several geometrical elements, each with a different size and shape. In this work, the variability of the parameters required to define each geometry has been set as random, but any other distribution could have been adopted. This methodology has been used to design five cubic scaffolds with interconnected pore channels that range from 200 to $800 \mu \mathrm{m}$ in diameter, each with an increased complexity of the internal geometrical arrangement. A clinical case study, consisting of an integration of one of these geometries with a craniofacial implant, is then presented.
\end{abstract}

Keywords: computer-aided design, bone tissue-engineering scaffolds, scaffold manufacturing, automated design methodology

\section{INTRODUCTION}

Tissue Engineering (TE) is an interdisciplinary field that applies the principles of engineering and the life sciences toward the development of biological substitutes that restore, maintain, or improve tissue function [1]. One approach to promote the regeneration of new tissue involves using scaffolds to provide a template to which cells can adhere, proliferate, and migrate, thus promoting the vascularization and the regeneration of the new tissue $[2,3]$. For this purpose, the scaffolds' internal structure plays an

*Corresponding author: Wolfson School of Mechanical and Manufacturing Engineering, Loughborough University, Loughborough, Leicestershire, LE11 3TU, UK. email: e.ramin@lboro. ac.uk essential role, in particular porosity, pore size, and pore interconnectivity $[\mathbf{4}, \mathbf{5}]$. In vitro tests show that inappropriate structures result in a lack of oxygen and nutrient provision, thus affecting the depth of penetration of new cells inside the scaffold. This depth of penetration is often less than $500 \mu \mathrm{m}$ for foam structures, such as those obtained using conventional scaffold fabrication techniques, that produce process-dependent architectures that are incapable of precisely controlling pore size and shape, porosity, and their spatial distributions, within the scaffold volume [6, 7]. Cells that colonize the periphery of the scaffold act as a barrier for a deeper diffusion of oxygen and nutrients, preventing new cells from migrating further and therefore a deeper vascularization. 
Since the regeneration of new tissue is affected by the internal architecture of such templates, a methodology to control pore size, shape, and location precisely is essential to promote and control the formation of new tissue.

The advent of layer-manufacturing (LM) technologies has overcome many limitations related to both the design and the fabrication of TE scaffolds and most importantly has made the two processes independent. This means that the design is not constrained to one specific fabrication process, allowing the design to be investigated as a separate field apart from the actual manufacturing process adopted.

In particular, these technologies allow the fabrication of implants with customized global anatomical shape, via combination with reverse engineered data from computed tomography (CT) or magnetic resonance imaging, with virtually no limits on the complexity of the geometry achievable. In addition, reproducible and irregular internal structures are obtainable, giving control over pore size, shape, interconnectivity, and porosity. The design of the global and internal architectures of scaffolds can be performed and precisely controlled within any conventional three-dimensional (3D) computer-aided design (CAD) software, a characteristic that may improve the flow of essential nutrients across the scaffold geometry, thereby aiding a deeper regeneration of new tissue [8-11].

Several research efforts have been made for designing TE scaffolds with controlled architecture [12-15]. A commonly adopted approach consists of selecting a unit cell from a library of predesigned unit cells, each of which has a predefined internal structure. The selected unit cell is then repeated in the three directions to match the size of the associated implant, whose overall geometry is usually derived from reverse engineered data. By means of CAD Boolean operations, the two geometries are merged to obtain the final architecture. In other studies [16-18], instead of using a predefined library of unit cells, topology optimization was utilized to design unit cells that matched different mechanical bone properties with given constraints in porosity.

This approach presents several drawbacks, such as limited gradients of pore size and shape, porosity, and lack of irregularities, unlike the features observed in natural trabecular bone structure [19]. In addition, the need for CAD Boolean operations makes the process particularly inefficient when the geometries to be merged become very complex and irregular, as in such cases the amount of computa- tional time, resources, and file size increase enormously [20]. As a result, the scaffold architectures are often limited to regular primitive shapes in the form of patterned straight lines, or square or circular holes [21].

The aim of the study was to develop an alternative design technique within a CAD environment that was able to integrate efficiently the complex design irregularities and geometrical gradients that feature in scaffold architectures. This was achieved by avoiding the use of Boolean operations and by introducing the concept of control and automation in the design process, which makes it much more flexible and efficient.

\subsection{TE scaffold requirements}

There are several requirements for TE scaffolds [22, 23] and, in particular, the following.

1. They must have high porosity and interconnecting pores of appropriate size and curvature. The optimal architecture should mimic that of the original tissue.

2. They should be produced from biologically acceptable materials.

3 . They must possess adequate mechanical properties for given load-bearing conditions.

4. They must have a tailored external shape to fit anatomical defects.

5. They should be efficiently manufactured and have reproducible internal structures.

While much research has been carried out on the mechanical properties of TE scaffolds $[\mathbf{1 1}, \mathbf{1 6}-\mathbf{1 8}$, 24-26], there has been comparatively little consideration of the design requirements of these highly complex geometries. Ideally, the optimal architecture for TE scaffolds should be the same as that of the native tissue, but few specific requirements have been quantified. For bone TE some data about porosity and pore size range are available, while data regarding pore shape, interconnectivity, and pore distributions are still unknown. Previous studies have suggested that the minimum pore size to be considered for mineralized bone regeneration should be about $100 \mu \mathrm{m}$ owing to cell size, migration requirements, and transport, although a minimum of about $300 \mu \mathrm{m}$ is recommended to enhance new bone formation and capillaries $[\mathbf{2 7}, \mathbf{2 8}]$. An upper limit for pore size is not explicitly defined as it is generally dictated by constraints associated with mechanical properties. However, other work has suggested a highest value of $800 \mu \mathrm{m}$ [28]. 
Similarly, minimum and maximum limits for porosity have not been suggested, but a value in the range of 30 to 90 per cent is usually adopted, depending on the material, process, and scaffold architecture [28]. In addition, porosity is not a particularly useful quantity as it only measures the total pore volume and does not provide any information as to how this is distributed throughout the scaffold [29].

This lack of detailed data makes it very difficult to optimize the structure of scaffolds, manufacturing process, and materials [29-31]; hence there is a need for a flexible design methodology that would allow the introduction of new qualitative and quantitative requirements whenever they become available.

\subsection{Trabecular bone structure}

In addition to flexibility, an ideal TE scaffolds design methodology should be able to represent highly complex and irregular geometries, such as those found in the human trabecular bone [19]. These requisites can be satisfied by the introduction of automation into the design process.

In order to assess and analyse these microstructures, several studies have been conducted using non-destructive techniques such as micro computed tomography $(\mu \mathrm{CT})[\mathbf{3 2}, 33]$ that are capable of acquiring images of small bone samples with resolutions of $1 \mu \mathrm{m}$ [34]. An example of trabecular bone structure acquired by $\mu \mathrm{CT}$ is depicted in Fig. 1, which shows the high degree of complexity in natural bone structures and, therefore, ideal bone TE scaffolds should possess a similar level of irregularities. However, the manual design of a large number of irregular features is an extremely time-consuming process, with scarce efficiency and flexibility, since it would require a very large amount of time to produce only one singular design [21]. Given that customized design is integral to a high proportion of tissue scaffolds, it can be appreciated that their manual design presents a crucial bottleneck.

Automation in the design process is the key factor to design a large number of irregular geometries in a short time, while maintaining a high level of flexibility.

\subsection{Efficient and flexible automated design}

The novelty of the methodology proposed in this work is formulated around a dedicated library of efficient routines, which have been developed in order to interact with advanced CAD software, and

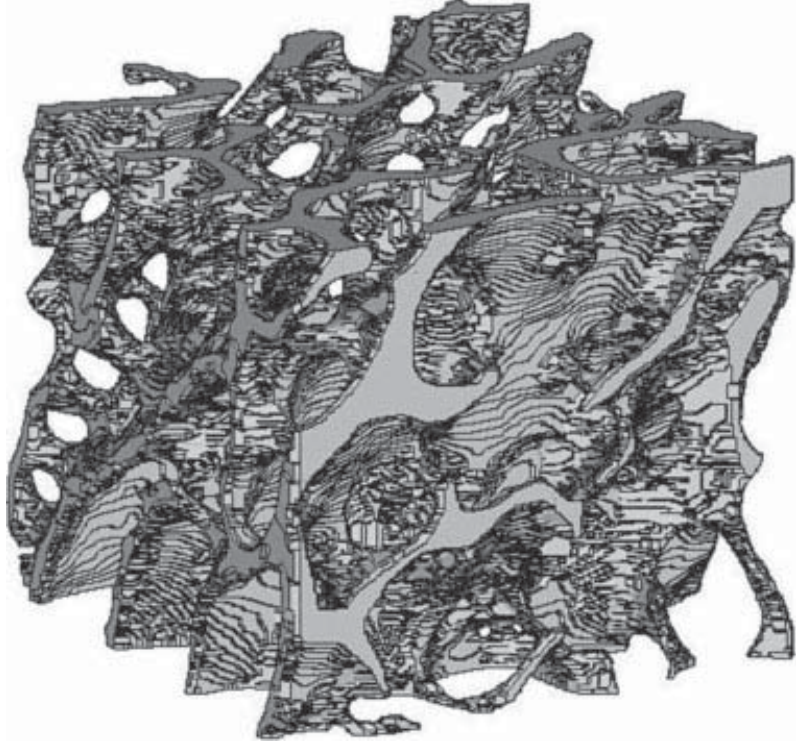

Fig. 1 Example of an STL representation of human trabecular bone structure from a $\mu \mathrm{CT}$ scan of a femoral neck

to perform the automatic design of the desired geometrical elements, each with its own different characteristics and parameters.

Geometrical parameters can be varied within userspecified ranges and in different ways, either randomly, or according to given mathematical functions, leading to infinite different combinations of the elements and thus flexibility in future design paradigms. Further flexibility can be achieved by introducing new variables and geometrical features, not considered in the current set of routines, by making few changes in the source code of the existing routines, as they have been written as a set of independent functions that act on given geometrical elements, without affecting the rest of the code.

Flexibility is therefore achieved by both expanding and modifying the existing set of functions, which constitutes a library of routines that has been written to interact with advanced 3D CAD software with powerful automation capabilities, allowing direct access to most of its internal classes through standard interfaces.

The classes can be accessed in several ways, depending on the CAD application. It can be done by including specific header files (.h) or 'dynamic link libraries' (.dll) files written with an 'application programming interface' (API). In all cases, an external application can access the original classes of the CAD software, thereby instructing it to design the required geometries and to perform other activities, without any manual intervention from the 
user. When possible, inheritance will even allow the expansion of the original classes, by adding new characteristics and functions. Hence the term 'advanced' CAD software, used to denote commercially available $\mathrm{CAD}$ applications that allow complete access to the modelling commands through its classes.

Furthermore, more efficient usage of computer memory can be achieved through automated design by removing all unnecessary operations such as opening windows to insert or modify data, selection and deselection of features, creation of primitive geometries, etc.

In some cases, automated saving operations can further reduce the amount of memory utilized by the application, as they empty the 'undo' buffer for the previous commands, thus occupying a large portion of memory when the number of operations becomes larger.

\section{MATERIALS AND METHODS}

The basic element that was utilized throughout this work was a multi-section solid. This element was initially chosen in order to analyse and refine the methodology, although any other geometry could have been chosen instead. The number of crosssections adopted was one or two, depending on the case, while the path was defined by a line or a curve in space. An example of a curved multi-section solid made of two sections is depicted in Fig. 2. The multisection solid can also be represented in its reverse form. In this case it forms a channel with the same shape inside a reference volume. Channels are utilized to represent the scaffold structure, while the solid representation is used for the theoretical shape of the regenerated tissue, assuming that the channels of the scaffold act as an ingrowth template for the regeneration of the new tissue.

Several routines were written for the presented cases, which can be grouped as, first, routines for defining the pore shape and size, second, routines for defining a 3D path for each channel, and, third, routines for designing the multi-section solid. Each routine was called by a 'main' program, which included other common data such as pore size range, position of each channel, number of channels in each directions, and definition of the reference volume.

The classes required in these routines were those defining two-dimensional (2D) and 3D primitives and curves, and those defining solid 3D elements. In addition, to optimize the memory usage, the

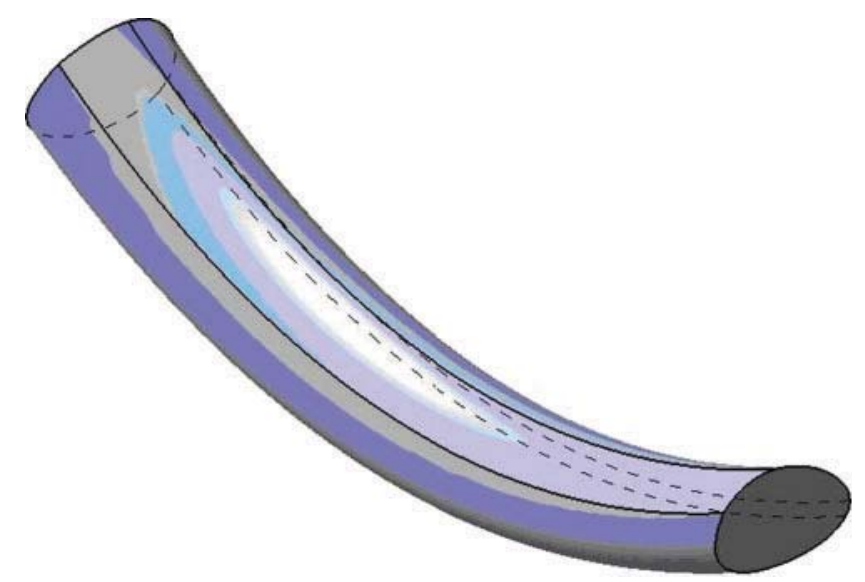

Fig. 2 Example of a multi-section solid adopted in this work

class defining the CAD file was used in the 'main' routine.

For example, for designing the pore shape, the class '2D spline' was used, together with some of its parameters such as tangents, curvature, and definition of control points. To create the control points, the class '2D point' was used, with other parameters such as the two coordinates of the points and the reference plane, which involved the use of the class 'plane'. Similarly, for designing the 3D path, the classes '3D point', '3D line', and '3D spline' were used, to define respectively the control points of a 3D spline and the starting and ends of a 3D segment. Finally, for designing the solid channel, the 'multisection solid' class was used, which required the specification of a number of $2 \mathrm{D}$ cross-sections together with a 3D curve for defining its spatial arrangement.

In order to test the feasibility of the proposed automated design, several case studies have been investigated. These examples show a progressive increase in geometry variables and complexity, related to an increasing number of design parameters and routines in accordance with the input data set by the user. The efficiency of such routines is assessed by two critical output parameters; computational time and the resultant file size. At this stage, the file size was used only to ascertain that it was within reasonable limits for use on a standard computer in both its CAD and Standard Tessellation Language (STL) formats. The reason for this is that, in cubic volumes, a pure manual versus automated design comparison will result in the same file size, unless a Boolean operation is required in the manual design, which occurs when the scaffold geometry has to be adapted to an irregular volume such as an implant. In these cases, which will be presented in 
future work, the file size can be significantly reduced by the introduction of automation in the integration process.

However, the design time required by each individual geometry provided an estimated comparison of the amount of time saved with respect to an average manual design. These examples consisted of a small cube $(10 \mathrm{~mm} \times 10 \mathrm{~mm} \times 10 \mathrm{~mm})$ consisting of differently oriented channels of various shapes and sizes. There was no particular requirement to use a cubic shape; any other reference geometry could have been used, but this shape provided a regular volume to direct attention to given parameters, apart from the location of each channel. In addition, this shape is useful to compare these scaffolds with cubic $\mu \mathrm{CT}$ samples of real trabecular bone, in further work.

All the structures reported in this work have a regular location and therefore the position of all channels are fixed. This means that the networks shown are irregular in terms of geometry, but not in terms of position. The location of each channel constitutes a parameter, which, like all other parameters, can be changed either randomly or according to any mathematical function. This was not considered in this section of the work since it was not required to assess the proposed methodology. This parameter will be studied in further work, in order to refine irregular distributions further.

In this work, all the input parameters have been altered randomly, each within a user-defined range, in order to increase the variability of the geometry.

The structures presented here were designed using Catia $^{\circledR}$ V5 R16 (by Dassault Systèmes (http://www. 3ds.com)) CAD software, which allowed full access to the required classes defined in several 'dll' files, and a standard Intel ${ }^{\circledR}$ Pentium ${ }^{\circledR} \mathrm{M}$ processor $1.60 \mathrm{MHz}$ computer, with $752 \mathrm{MB}$ of random-access memory. A standard computer has been used in this work to pursue novel automated design of TE scaffolds using moderate computation abilities, and thus to avoid the need for high-performance computers.

A clinical implant study, consisting of a craniofacial implant, is also introduced. The proposed design methodology is evaluated in this case in combination with one of the scaffold designs presented. The digital model of the craniofacial implant was derived from CT scan data. From the data, the Initial Graphics Exchange Specification (IGES) and STL files of the implant were generated.

\subsection{Evaluation of pore characteristics}

In order to achieve different and irregular pore shapes in the cross-sections of each channel, closed 2D splines consisting of four control points, were used. Each control point was positioned as a vertex of a square and, in the first approximation, all pore sizes were estimated on the basis of the side of each square and its area. The actual porosity is greater than this value and can be computed using the mathematical formulation of Catia spline curves. This approximation has been used for defining an equivalent diameter of the pore, as in literature the size of the pore is often reported as the 'diameter', without any other specific data regarding the real pore shape. In addition, a more precise computation would not affect the design methodology itself. Figure 3 gives a brief example of how the algorithm for generating such 2D splines works. The estimated pore size is defined by the highlighted square, while

\section{STEPS TO CONTROL PORE SLKE AND SHAPE:}

- Set pore size ramge (exanqle 0.2 to $0.8 \mathrm{~nm}$ )

- Generation of a random roumber withm this range ( $0.15 \mathrm{~nm}$ in this example)

- Creation of 5 cortol points (1st and Sth coincident and equal tamgert) positioned as 4 vertices of an ideal square

- To obtain different shapes, the tamgents are altered

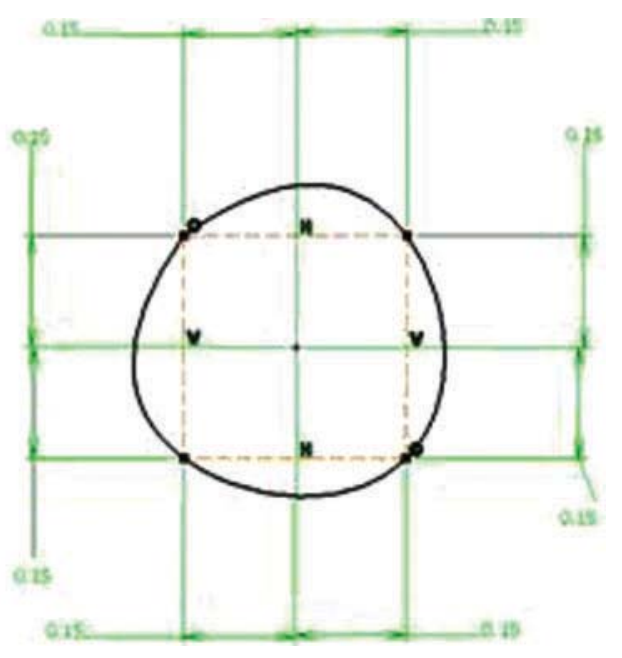

Fig. 3 Steps for designing different pore size and shape in the 2D cross-sections 
the actual size is the area enclosed by the spline. It is possible to define any other design layout by using, for example, a different number of control points in which the coordinates do not necessarily form the vertices of a square but may be varied randomly within different ranges. It is also possible to control the wall thickness between two consecutive holes, setting the minimum and maximum and changing its value, or to introduce other parameters such as the curvature at each control point. The presented layout was chosen for its natural definition of equivalent pore diameter. Finally, the position of a given pore was set as the centre of each element of a grid formed by $n \times n$ squares, $n$ being the number of channels.

\section{RESULTS}

\subsection{Case 1}

This case presents a regular distribution of straight and orthogonal channels with different shapes, obtained by randomly changing the value of the tangents at the control points. The pore size range was set from $0.2 \mathrm{~mm}$ to $0.8 \mathrm{~mm}$, although the actual pore size is slightly greater, since it was estimated considering the square area formed by the four control points defining each cross-section. The pore size range chosen is suitable for osteoregeneration. The scaffold and complementary geometry are illustrated in Figs 4(a) and (b) respectively. The details of this case are reported in Table 1.

\section{2 $\quad$ Case 2}

This case presents a regular distribution of straight and orthogonal channels, with step gradients in the cross-sections. In each pore, the shape changes every $2 \mathrm{~mm}$ by randomly altering the tangents at the

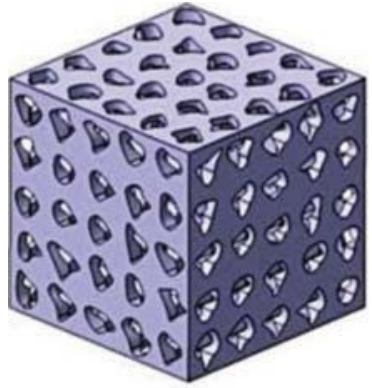

(a)

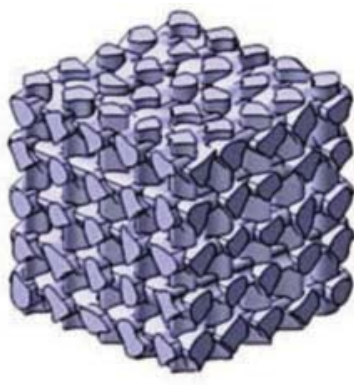

(b)
Fig. 4 Case 1: (a) scaffold; (b) theoretical regenerated bone
Table 1 Case 1 scaffold, main input and output parameters

\begin{tabular}{|c|c|}
\hline Parameter & Value \\
\hline \multicolumn{2}{|l|}{ Input parameters, constants } \\
\hline Cube side & $10 \mathrm{~mm}$ \\
\hline Pore size & $\begin{array}{l}\text { Range set from } 0.2 \mathrm{~mm} \text { to } \\
0.8 \mathrm{~mm}\end{array}$ \\
\hline Location of pores & Regularly fixed \\
\hline Number of pores & 5 \\
\hline \multicolumn{2}{|l|}{ Input parameters, variables } \\
\hline Tangents at four control points & Random $\times 1$ section \\
\hline \multicolumn{2}{|c|}{ sketch, point2D, line2D, pad, pocket, controlPoint2D, spline2D } \\
\hline \multicolumn{2}{|l|}{ Output parameters } \\
\hline Computational time & $13 \min$ \\
\hline Average time per channel & $10.4 \mathrm{~s}$ \\
\hline Porosity & $55.27 \%$ \\
\hline Catia file size & $10.7 \mathrm{MB}$ \\
\hline STL size & $9.95 \mathrm{MB}$ \\
\hline
\end{tabular}

four control points. As in the previous case, the approximate pore size range was set from $0.2 \mathrm{~mm}$ to $0.8 \mathrm{~mm}$. Figure 5 shows some step changes in the cross-sections in more detail. The main data from this case are summarized in Table 2.

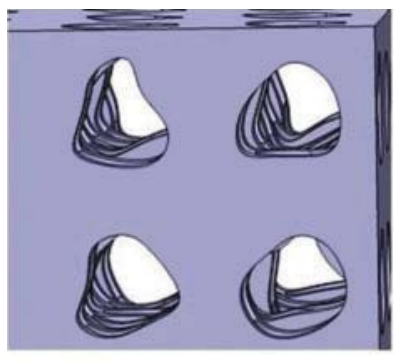

(a)

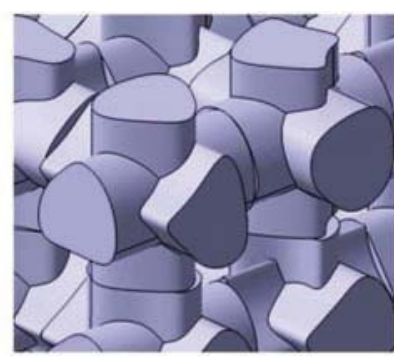

(b)
Fig. 5 Case 2: details of the step changes in different cross-sections

Table 2 Case 2 scaffold, main input and output parameters

\begin{tabular}{ll}
\hline Parameters & Value \\
\hline $\begin{array}{l}\text { Input parameter, constant } \\
\text { Cube side } \\
\text { Pore size }\end{array}$ & $\begin{array}{l}10 \mathrm{~mm} \\
\text { Range set from } 0.2 \mathrm{~mm} \text { to } \\
0.8 \mathrm{~mm} \\
\text { Regularly fixed }\end{array}$ \\
$\begin{array}{l}\text { Location of pores } \\
\text { Number of pores }\end{array}$ & 5 \\
$\begin{array}{l}\text { Step distance } \\
\text { Input parameters, variables }\end{array}$ & $2 \mathrm{~mm}$ \\
$\begin{array}{l}\text { Tangents at four control points } \\
\text { Input parameters, Catia classes } \\
\text { sketch, point2D, line2D, pad, pocket, controlPoint2D, spline2D, }\end{array}$ & Random $\times 5$ sections \\
$\quad$ hybridShapePlaneOffset & \\
$\begin{array}{l}\text { Output parameters } \\
\text { Computational time }\end{array}$ & $15 \mathrm{~min}$ \\
Average time per channel & $12 \mathrm{~s}$ \\
Porosity & $49.95 \%$ \\
Catia File size & $15 \mathrm{MB}$ \\
STL size & $16.9 \mathrm{MB}$
\end{tabular}




\subsection{Case 3}

This case presents a regular distribution of straight and non-orthogonal channels with two cross-sections of different shape. The linear path of each channel was defined by randomly connecting two different central points, located on opposite faces of the cube. The number of pores has been increased from 5 to 7 to study the effects of adding a few more channels on the computational time and file size, which were negligible.

As in the other cases, pore shape was changed randomly by controlling the tangents at the control points. Figure 6 shows more details of some linear channels. The main data of this case are summarized in Table 3.

\subsection{Case 4}

This case presents a regular distribution of curved channels with two cross-sections of different shape,
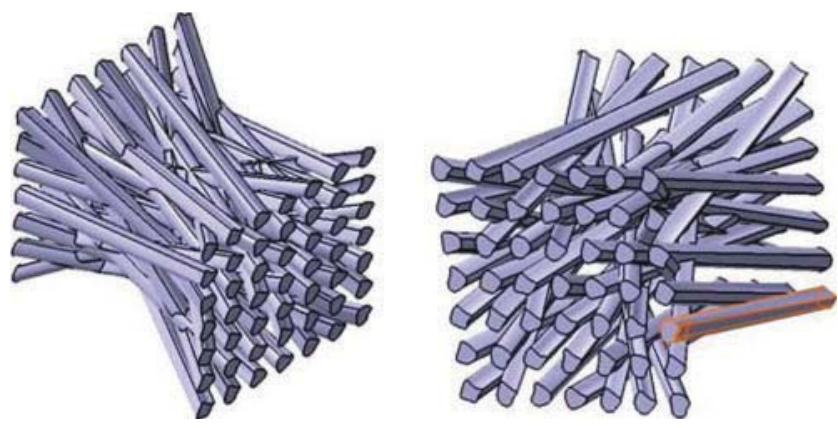

Fig. 6 Case 3: details of some linear channels

Table 3 Case 3 scaffold, main input and output parameters

\begin{tabular}{|c|c|}
\hline Parameter & Value \\
\hline \multicolumn{2}{|l|}{ Input parameters, constants } \\
\hline Cube side & $10 \mathrm{~mm}$ \\
\hline Pore size & $\begin{array}{l}\text { Range set from } 0.2 \mathrm{~mm} \text { to } \\
0.8 \mathrm{~mm}\end{array}$ \\
\hline Location of pores & Regularly fixed \\
\hline Number of pores & 7 \\
\hline \multicolumn{2}{|l|}{ Input parameters, variables } \\
\hline Linear path for each channel & $\begin{array}{l}\text { Random association of the } \\
\text { central points of two cross- } \\
\text { sections in opposite planes }\end{array}$ \\
\hline Tangents at four control points & Random $\times 2$ sections \\
\hline \multirow{2}{*}{\multicolumn{2}{|c|}{$\begin{array}{l}\text { Input parameters, Catia classes } \\
\text { sketch, point } 2 \mathrm{D} \text {, line } 2 \mathrm{D} \text {, controlPoint } 2 \mathrm{D} \text {, spline } 2 \mathrm{D} \text {, hybridShape- } \\
\text { PlaneOffset, hybridShapePointCoord, hybridShapeLinePtPt, hy- } \\
\text { bridShapeLoft }\end{array}$}} \\
\hline & \\
\hline \multicolumn{2}{|l|}{ Output parameters } \\
\hline Computational time & $20 \min$ \\
\hline Average time per channel & $8 \mathrm{~s}$ \\
\hline Porosity & $44.16 \%$ \\
\hline Catia file size & $35.7 \mathrm{MB}$ \\
\hline STL size & $17.4 \mathrm{MB}$ \\
\hline
\end{tabular}

converging to the centre of the volume. This arrangement sought to illustrate that a higher porosity and pore interconnectivity may be directed on a given convergence, utilizing the centre as an example. Convergence could be situated at a given location at single or multiple sites. This was intended to illustrate the flexibility of the proposed methodology, which can be adapted to include different hypotheses regarding particular geometrical distributions. The curved path of each channel was defined by a 3D spline, which connected two different sections at the same control points, through the cube centre. It is possible that, during this process, errors such as twisted and sharp geometries or cusps may cause the failure of the program and interrupt the design process. Subsequently, a routine was specifically designed to intercept run-time errors and to correct the geometry. This routine automatically detected any error in the geometry and altered the specified input parameters until it produced an error-free geometry without any manual intervention. The user can select which parameters have to be changed by this routine. By default, the tangents at the control points were modified, and subsequently the pore shape. This procedure increased the computational time, as it needed to calculate many different combinations of parameters in order to find a suitable geometry. However, the advantage consisted in the automated design of more complex channels, involving curved and multi-section solids.

Figure 7 shows some curved paths and their convergence to the scaffold centre in more detail. The main data of this case are summarized in Table 4 .

\subsection{Case 5}

This case relates to case 4 , although the network is arranged differently. The channels no longer converge to the centre and instead, their path is randomly set. The $3 \mathrm{D}$ spline that defines the path
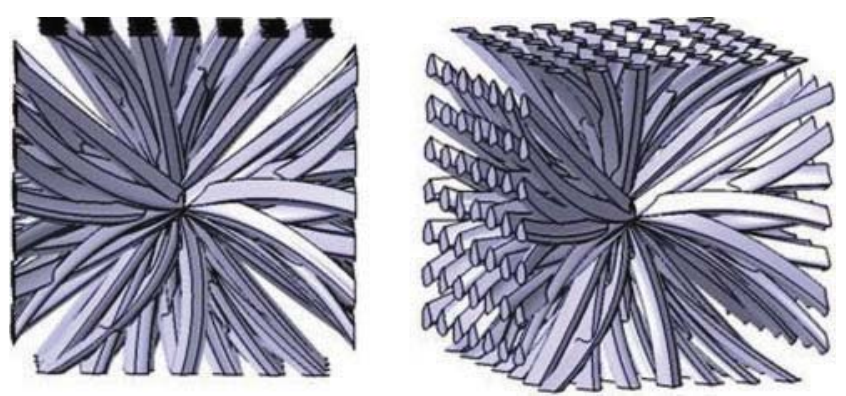

Fig. 7 Case 4: details of some curved paths and their convergence to the scaffold centre 
Table 4 Case 4 scaffold, main input and output parameters

\begin{tabular}{|c|c|}
\hline Parameter & Value \\
\hline \multicolumn{2}{|l|}{ Input parameters, constants } \\
\hline Cube side & $10 \mathrm{~mm}$ \\
\hline Pore size & $\begin{array}{l}\text { Range set from } 0.2 \mathrm{~mm} \text { to } \\
0.8 \mathrm{~mm}\end{array}$ \\
\hline Location of pores & Regularly fixed \\
\hline Number of pores & 7 \\
\hline \multicolumn{2}{|l|}{ Input parameters, variables } \\
\hline Curved path for each channel & $\begin{array}{l}\text { Random association of the } \\
\text { central points of two cross- } \\
\text { sections in opposite planes, } \\
\text { connected by a 3D curve } \\
\text { through the centre }\end{array}$ \\
\hline Tangents at four control points & Random $\times 2$ sections \\
\hline \multicolumn{2}{|l|}{ Input parameters, Catia classes } \\
\hline \multicolumn{2}{|c|}{$\begin{array}{l}\text { sketch, point2D, line2D, controlPoint2D, spline2D, hybridShape- } \\
\text { PlaneOffset, hybridShapePointCoord, hybridShapeSpline, } \\
\text { hybridShapeLoft }\end{array}$} \\
\hline \multicolumn{2}{|l|}{ Output parameters } \\
\hline Computational time & $20 \min$ \\
\hline Average time per channel & $8 s$ \\
\hline Porosity & $33.56 \%$ \\
\hline Catia file size & $20.6 \mathrm{MB}$ \\
\hline STL size & $13.6 \mathrm{MB}$ \\
\hline
\end{tabular}

for each channel is made up of three points, the first and the third of which are in the two cross-sections (and correspond to two control points). The coordinates of the middle point are randomly variable within a range dictated by user input. In this case, a range of \pm 20 per cent of the length of the cube was defined, which meant $\pm 2 \mathrm{~mm}$ in each direction, with respect to the cube centre, although of course any intermediate value could have been imposed. As in the previous case, a routine to detect run-time errors and automatically to correct the geometry was necessary. The parameters modified by this routine were the tangents at the control points of the 2D splines and the pore size. Figure 8 shows some curved paths and their random connections in more detail. The main data from this case are summarized in Table 5.
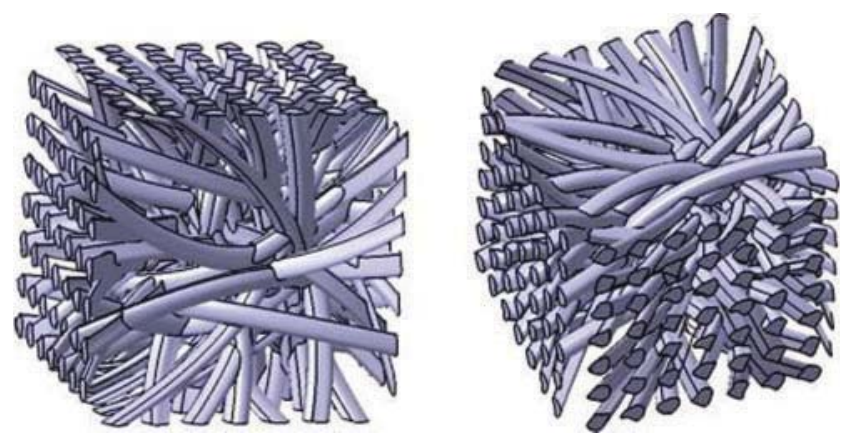

Fig. 8 Case 5: details of some curved paths and their random connections
Table 5 Case 5 scaffold, main input and output parameters

\begin{tabular}{|c|c|}
\hline Parameter & Value \\
\hline \multicolumn{2}{|l|}{ Input parameters, constants } \\
\hline Cube side & $10 \mathrm{~mm}$ \\
\hline Pore size & Range set from $0.2 \mathrm{~mm}$ to $0.8 \mathrm{~mm}$ \\
\hline Location of pores & Regularly fixed \\
\hline Number of pores & 7 \\
\hline $\begin{array}{l}\text { Control point of the two } \\
\text { cross-sections to be } \\
\text { connected by the } 3 \mathrm{D} \\
\text { curve }\end{array}$ & Fixed \\
\hline Ideal central volume & $\begin{array}{l} \pm 20 \% \text { of the length of the cube in } \\
\text { each direction }\end{array}$ \\
\hline \multicolumn{2}{|l|}{ Input parameters, variables } \\
\hline $\begin{array}{l}\text { Curved path for each } \\
\text { channel }\end{array}$ & $\begin{array}{l}\text { Random association of the central } \\
\text { points of two cross-sections in } \\
\text { opposite planes, connected by a } \\
\text { 3D curve through a random } \\
\text { point within the ideal central } \\
\text { volume }\end{array}$ \\
\hline \multicolumn{2}{|c|}{ Tangents at four control pointsRandom $\times 2$ sections } \\
\hline \multicolumn{2}{|c|}{ Input parameters, Catia classes } \\
\hline \multicolumn{2}{|c|}{$\begin{array}{l}\text { sketch, point2D, line2D, controlPoint2D, spline2D, hybridShape- } \\
\text { PlaneOffset, hybridShapePointCoord, hybridShapeSpline, } \\
\text { hybridShapeLoft }\end{array}$} \\
\hline \multicolumn{2}{|l|}{ Output parameters } \\
\hline Computational time & $22 \mathrm{~min}$ \\
\hline Average time per channel & $9 \mathrm{~s}$ \\
\hline Porosity & $36.77 \%$ \\
\hline Catia file size & $35.5 \mathrm{MB}$ \\
\hline STL size & $24 \mathrm{MB}$ \\
\hline
\end{tabular}

\subsection{Clinical case study}

The experimental cases have shown that it is possible to achieve a flexible and efficient automated design of regular distributions of irregularly and randomly connected channels. The advantages in terms of time, when compared with manual design, are evident when, instead of considering relatively small samples, a volume of the size of an implant such as the example in Fig. 9 is required. In general, the larger and more complex the implant, the more advantageous is the automated design. In this particular clinical case study, the size of the implant was $51 \mathrm{~mm} \times 40.6 \mathrm{~mm} \times 8.55 \mathrm{~mm}$ and consisted of a regular distribution of 420 case 4 channels in the $X-Y$ plane. Each channel had two cross-sections, and therefore the number of random parameters was 26880 , given that each of the 420 channels had eight control points and eight tangents. The manual modelling of such a network would be an extremely arduous task, since for each channel the user would need to find random combinations of parameters that would not lead to cusps, twists, and other errors in the geometry - features that may occur frequently in non-linear arrangements. Instead, the design time taken by the case 6 routine was $30 \mathrm{~min}$, with an average time of $4.3 \mathrm{~s}$ per channel. The same computational resources as previously described were 

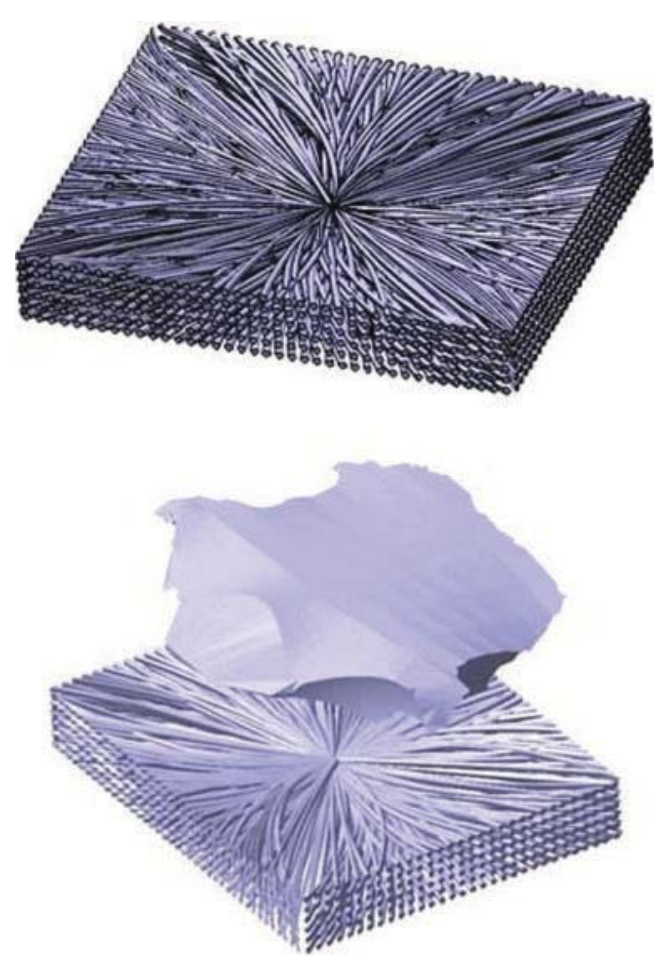

Fig. 9 Case 4 structure extended to match the geometry of a craniofacial implant

used, and the resultant Catia file size was $78.8 \mathrm{MB}$. This network of channels was modelled only in the $X$ and $Y$ directions, as it was associated with a craniofacial implant - essentially a flat bone, although the network along the $Z$ axis could have been included.

\section{DISCUSSION}

The development of a library of routines in advanced CAD software allows an efficient and flexible automated design. The slowest routine showed an average computational time of $12 \mathrm{~s}$ to design 75 straight channels, while the fastest reached an average of $8 \mathrm{~s}$ for 147 channels. In the clinical case study, consisting of 420 channels, the average design time was only $4.3 \mathrm{~s}$. These results cannot be directly compared because, as they were made of random geometries, the time will be affected by each particular combination of parameters. In addition, when curved channels are present, the computational time will include the time required to detect and correct any error in the geometry, the type and number of which will be different at every run, depending on the random combinations of the parameters. However, these results can be compared with an average time required to design similar structures manually, showing that there is a huge saving of time in the design process by introducing automation. In the worst-case scenario (fastest manual design time versus slowest automated design time), a CAD manual design can be estimated in between $45 \mathrm{~s}$ to several minutes per channel, while the slowest routine was around $12 \mathrm{~s}$, showing a minimum time saving of 75 per cent, which became more than 90 per cent for the clinical case study. In addition, the estimated manual design supposed that a human operator is capable of performing an uninterrupted design for many consecutive hours at once, which is an unrealistic ideal scenario.

As anticipated in section 2, there is no significant reduction in file size because, for regular volumes, CAD Boolean operations are not involved.

However, these are not the only advantages given by automation, as it would also allow the study of the influence of specific geometric parameters on tissue regeneration, such as the shape of channels and curvature, provided that the geometries represented would be fully reproducible by an LM system. The manufacturing aspects of these scaffolds were not considered at this stage, as the attention was focused on addressing the main issues relating to the design. However, these aspects will be considered as part of future work.

Another advantage is that the values of all the random parameters can be stored in external files and used, for instance, as input to redesign similar structures and subsequently the random architectures obtained and their particular combination of parameters could be further investigated. Furthermore, instead of a random change, any mathematical function can be set for all or some of the inputs in order to mimic, for instance, the different densities found in bone structures.

The possibilities to develop the library of routines further are infinite and overcome the lack of flexibility and the rigid schemes generally encountered in CAD software. The flexibility and versatility of this methodology permit adaptation and modification, when, for instance, the requirements for TE scaffolds will be better quantified. Figure 10 summarizes the steps followed by the algorithms proposed in this automated design process.

Step 1. The geometrical variables and constants, together with their ranges of variation, are defined.

Step 2. Automated design of the external shape, in this case a cube of $10 \mathrm{~mm}$ side, is carried out. 


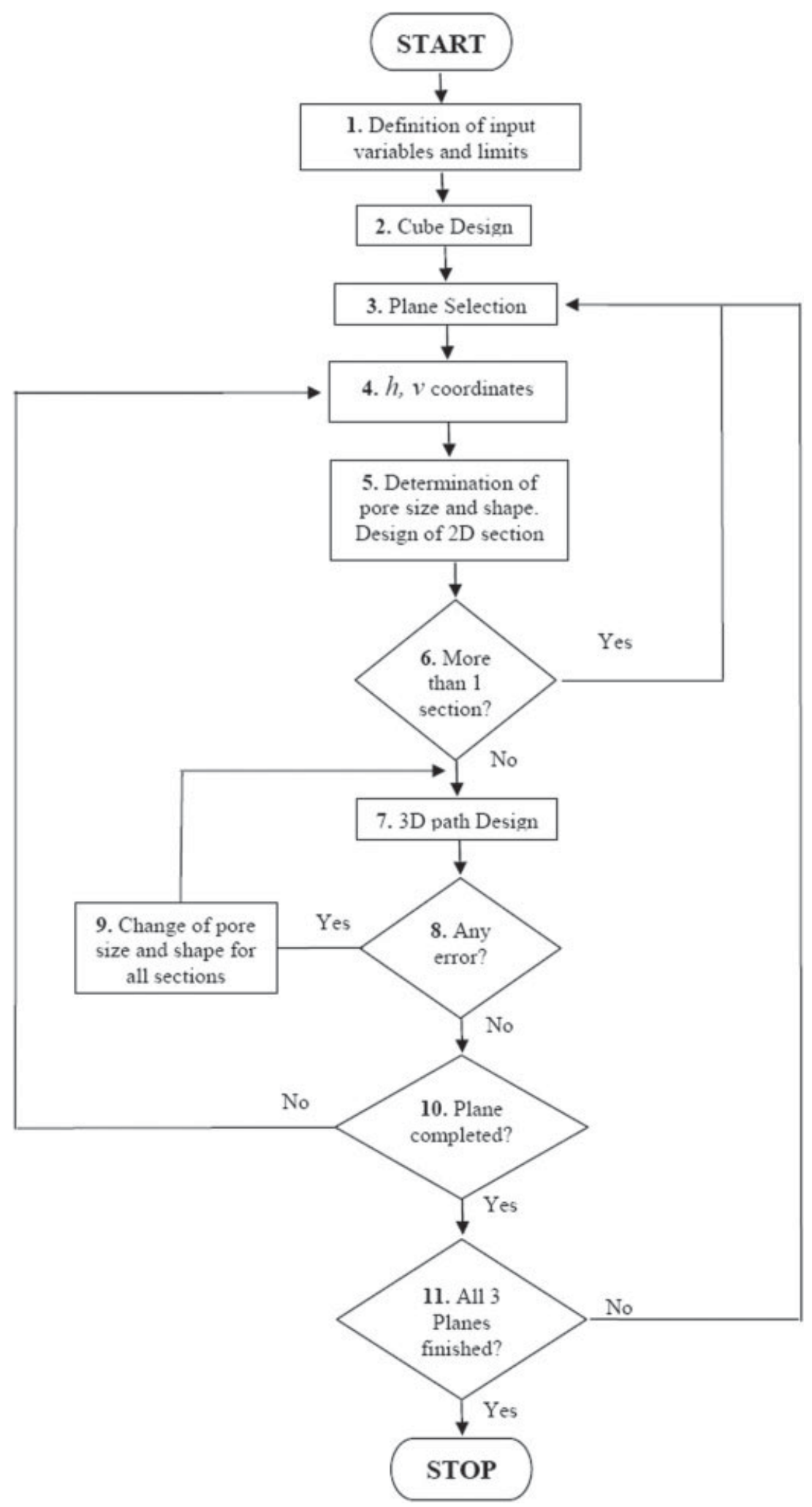

Fig. 10 Summary of the proposed automated design process

Step 3. The planes where the cross-sections are drawn are selected. This selection is performed automatically according to the geometrical variables defined in step 1 above. In the cases proposed here, only opposite faces of the cube were connected.

Step 4. The planar coordinates of the centre of each cross-section are computed. 
Step 5. The pore shape and size are designed and determined according to both the input variables defined in step 1 and their variability. In the proposed cases a random variation was set for both pore size and shape.

Step 6. If the channel is made of more than one cross-section, steps 3 to 5 are repeated for the given number of curves.

Step 7. The 3D arrangement of the channel is defined and the geometry designed.

Step 8. If any error occurs, the pore size and shape are changed.

Step 9. Eventually the 3D path is redefined and the new geometry designed. Steps 7,8 , and 9 are repeated until there are no other errors in the geometry.

Steps 10 and 11. The previous steps are repeated until all the cross-sections on all the selected planes are completed.

A drawback encountered at this stage, however, consisted in using Boolean operations to merge the two structures depicted in Fig. 9, as they become very inefficient when the geometries are complex and irregular. In such cases, these operations are time-consuming, require many computational resources, and drastically increase the file size. Furthermore, if a routine controls the pore size and shape in the boundary box, the actual pore size and shape will be modified by the merging process, as they will depend on where each channel intersects the surfaces of the implant. In order to overcome this loss of control in the actual geometry, an efficient merging methodology is needed that would not require the use of any Boolean operation and would perform the automated design directly on the implant geometry, without the need for any other reference volume. This aspect will be investigated further and presented in future work.

\section{CONCLUSIONS}

Even though it was proven that, through the development of a specific library of routines in advanced CAD, important drawbacks in the design of complex and irregular TE scaffolds can be overcome, some factors are not yet incorporated in this methodology. The geometrical design features of the progressive case studies focus on the currently defined biological requirements identified in other studies. However, there is a clear lack of quantitative and qualitative data regarding the requirements for TE scaffolds and the structures presented in this work were designed according to the data available in the literature. Therefore, in order to design biomimetic TE scaffolds, more quantitative data are required in terms of pore distribution, size, and shape, as well as porosity. In addition, the proposed methodology needs to be further developed to include an efficient integration process that would not involve the use of any CAD Boolean operation when the design is carried out on to a general irregular volume. Finally, at this stage, the manufacturing aspects have not been considered, primarily in terms of resolution, materials, and reproducibility, although there are a great number of researchers engaged in this pursuit worldwide. This work is fundamental and complementary to these activities.

\section{FUTURE WORK}

The automated design proposed in this work constitutes the initial experiments of a $\mathrm{PhD}$ research project aiming to develop an efficient and automated design methodology for biomimetic geometries using advanced CAD methods. The project focuses on design but is likely to be of significant interest to other areas dependent on CAD representations such as simulation, modelling, or relevant manufacturing processes. Future work will involve addressing the lack of quantitative and qualitative data through the analysis of real human trabecular bone samples from $\mu \mathrm{CT}$ scan data. By the identification of more quantitative requirements, the presented design methodology will be refined in order to produce more complex and irregular biomimetic TE scaffolds, with pore size and porosity distributions similar to those found in the $\mu \mathrm{CT}$ samples analysed.

Finally, to complete the proposed design methodology, an efficient and automated integration process will be developed. In order to avoid the use of any Boolean operation, new routines will be added to the existing library, which would be able to perform the automated design on whatever irregular volume, such as those found in anatomical implants, instead of cubic volumes.

\section{REFERENCES}

1 Langer, R. and Vacanti, J. P. Tissue engineering. Science, 1993, 260, 920-926.

2 Mironov, V., Boland, T., Trusk, T., Forgacs, G., and Markwald, R. R. Organ printing: computer-aided 
jet-based 3D tissue engineering. Trends Biotechnol., 2003, 21(4), 157-161.

3 Capito, R. M. and Spector, M. Scaffold-based articular cartilage repair. IEEE Engng in Medicine Biology Mag., 2003, 22(5), 42-50.

4 Williams, J. M., Adewunmi, A., Schek, R. M., Flanagan, C. L., Krebsbach, P. H., Feinberg, S. E., Hollister, S. J., and Das, S. Bone tissue engineering using polycaprolactone scaffolds fabricated via selective laser sintering. Biomaterials, 2005, 26(23), 4817-4827.

5 Taboas, J. M., Maddox, R. D., Krebsbach, P. H., and Hollister, S. J. Indirect solid freeform fabrication of local and global porous, biomimetic and composite 3-D polymer-ceramic scaffolds. Biomaterials, 2003, 24(1), 181-194.

6 Sachlos, E. and Czernuszka, J. T. Making tissue engineering scaffolds work. Review on the application of solid freeform fabrication technology to the production of tissue engineering scaffolds. Eur. Cells Mater., 2003, 5, 29-40.

7 Tan, K. H., Chua, C. K., Leong, K. F., Naing, M. W., and Cheah, C. M. Fabrication and characterization of three-dimensional poly(ether-ether-ketone)-hydroxyapatite biocomposite scaffolds using laser sintering. Proc. IMechE, Part H: J. Engineering in Medicine, 2005, 219, 183-194.

8 Bartolo, P. and Bidanda, B. Bio-materials and prototyping applications in medicine, 2008 ch. 5, pp. 67-75 (Springer-Verlay, Berlin).

9 Sachlos, E., Reis, N., Ainsley, C., Derby, B., and Czernuszka, J. T. Novel collagen scaffolds with predefined internal morphology made by solid freeform fabrication. Biomaterials, 2003, 24, 14871497.

10 Sun, W., Starly, B., Nam, J., and Darling, A. Bio$\mathrm{CAD}$ modelling and its applications in computeraided tissue engineering. Computer-Aided Des., 2005, 37, 1097-1114.

11 Chu, G. T. M., Orton, D. G., Hollister, S. J., Feinberg, S. E., and Halloran, J. W. Mechanical and in vivo performance of hydroxyapatite implants with controlled architectures. Biomaterials, 2002, 23, 1283-1293.

12 Chua, C. K., Leong, K. F., Cheah, C. M., and Chua, S. W. Development of a tissue engineering scaffold structure library for rapid prototyping. Part 1: investigation and classification. Int. J. Advd Mfg Technol., 2003, 21(4), 291-301.

13 Naing, M. W., Chua, C. K., Leong, K. F., and Wang, Y. Fabrication of customised scaffolds using computer-aided design and rapid prototyping techniques. Rapid Prototyping J., 2005, 11 (4), 249-259.

14 Nam, J., Starly, B., Darling, A., and Sun, W. Computer aided tissue engineering for modelling and design of novel tissue scaffolds. ComputerAided Des. Applic., 2004, 1, 633-40.

15 Starly, B., Lau, W., Bradbury, T., and Sun, W. Internal architecture design and freeform fabrication of tissue replacement structures. ComputerAided Des., 2006, 38(2), 115-124.
16 Hollister, S. J., Lin, C. Y., Saito, E., Schek, R. D., Taboas, J. M., and Williams, J. M. Engineering craniofacial scaffolds. Orthodontics Craniofacial Res., 2005, 8(3), 162-173.

17 Lin, C. Y., Kikuchi, N., and Hollister, S. J. A novel method for biomaterial scaffold internal architecture design to match bone elastic properties with desired porosity. J. Biomechanics, 2004, 37(5), 623-636.

18 Hollister, S. J., Maddox, R., and Taboas, J. Optimal design and fabrication of scaffolds to mimic tissue properties and satisfy biological constraints. Biomaterials, 2002, 23, 4095-4103.

19 Uchiyama, T., Tanizawa, T., Muramatsu, H., Endo, N., Takahashi, H. E., and Hara, T. Threedimensional microstructural analysis of human trabecular bone in relation to its mechanical properties. Bone, 1999, 25(4), 487-491.

20 Cowin, S. C. Bone mechanics handbook, 2nd edition, 2001, ch. 36, pp. 1-5 (CRC Press, Boca Raton, Florida).

21 Starly, B. and Sun, W. Internal scaffold architecture designs using Lindenmayer Systems. Computer-Aided Des. Applic., 2007, 4, 395-403.

22 Das, S. and Hollister, S. J. Tissue engineering scaffolds. In Encyclopedia of materials: science and technology, 2003, pp. 1-7 (Elsevier, Amsterdam).

23 Liu, C. Z., Sachlos, E., and Wahl, D. A. On the manufacturability of scaffold mould using a 3D printing technology. Rapid Prototyping J., 2007, 13(3), 163-174.

24 Hutmacher, D. W., Schantz, T., Zein, I., Ng, K. W., Teoh, S. H., and Tan, K. C. Mechanical properties and cell cultural response of polycaprolactone scaffolds designed and fabricated via fused deposition modelling. J. Biomed. Mater. Res., 2001, 55(2), 203-216.

25 Charriere, E., Lemaitre, J., and Zysset, P. Hydroxyapatite cement scaffolds with controlled macroporosity: fabrication protocol and mechanical properties. Biomaterials, 2003, 24(5), 809-817.

26 Wang, H. V., Johnston, S. R., and Rosen, D. W. Design of a graded cellular structure for an acetabular hip replacement component. In Proceedings of the 17th Solid FreeForm Fabrication Symposium, Austin, Texas, USA, 14-16 August 2006, pp. 111-123 (University of Texas at Austin, Austin, Texas).

27 Grant, P., Tomlins, P., Khurana, M., Vadgamma, P., James, S. L., and Mikhalovsky, S. V. In-vivo usage of biomaterials: a snapshot of current activity. NPL Report MATC(A) 159, National Physical Laboratory, Teddington Middlesex, UK, 2003.

28 Karageorgiou, V. and Kaplan, D. Porosity of 3D biomaterial scaffolds and osteogenesis. Biomaterials, 2005, 26, 5474-5491.

29 Tomlins, P., Grant, P., Mikhalovsky, S., Mikhalovska, L., James, S., and Vadgama, P. Characterisation of polymeric tissue scaffolds, NPL Measurement Good Practice Guide, No.89, (National Physical Laboratory, Teddington, Middlesex). 
30 Willows, A., Fan, Q., Ismail, F., Vaz, C. M., Tomlins, P. E., Mikhalovska, L., Mikhalovsky, S., James, S., and Vadgama, P. Electrochemical assessment of tissue scaffold degradation. NPL Report DEPC-MPR-049, National Physical Laboratory, Teddington, Middlesex, UK, 2006.

31 Wahl, D. A., Sachlos, E., Liu, C., and Czernuszka, J. Controlling the processing of collagen-hydroxyapatite scaffolds for bone tissue engineering. $J$. Mater. Sci. Mater. Medicine, 2006, 18(2), 201-209.

32 Jones, A. C., Milthorpe, B., Averdunk, H., Limaye, A., Senden, T. J., Sakellariou, A., Sheppard, A.
P., Sok, R. M., Knackstedt, M. A., and Brandwood, A. Analysis of $3 \mathrm{D}$ bone ingrowth into polymer scaffolds via micro-computed tomography imaging. Biomaterials, 2004, 25(20), 49474954.

33 Downey, P. A. and Siegel, M. I. Perspectives - bone biology and the clinical implications for osteoporosis. Phys. Therapy, 2006, 86(1), 77-91.

34 Genant, H. K., Gordon, C., Jiang, Y., Lang, T. F., Link, T. M., and Majumdar, S. Advanced imaging of bone macro and micro structure. Bone, 1999, 25(1), 149-152. 